\title{
Enhancement of Processing Capabilities of Hippocampus Lobe: A P300 Based Event Related Potential Study
}

\author{
Neelesh Benet ${ }^{1,2}$, Rajalakshmi Krishna ${ }^{2}$, and Vijay Kumar ${ }^{1}$ \\ ${ }^{1}$ Department of Audiology and Speech-Language Pathology, Amity University Haryana, Gurgaon, India \\ ${ }^{2}$ All India Institute of Speech and Hearing, Mysuru, India
}

Received January 22, 2021

Revised April 5, 2021

Accepted April 8, 2021

\section{Address for correspondence \\ Vijay Kumar, MSc, PhD \\ Department of Audiology and \\ Speech-Language Pathology, \\ Amity University, Gurgaon, \\ Haryana 122413, India \\ Tel $+91-9650522584$ \\ Fax +91-0124-2337015 \\ E-mail vkumar@ggn.amity.edu}

Background and Objectives: The influence of music training on different areas of the brain has been extensively researched, but the underlying neurobehavioral mechanisms remain unknown. In the present study, the effects of training for more than three years in Carnatic music (an Indian form of music) on the discrimination ability of different areas of the brain were tested using P300 analysis at three electrode placement sites. Subjects and Methods: A total of 27 individuals, including 13 singers aged 16-30 years (mean \pm standard deviation, $23 \pm 3.2$ years) and 14 non-singers aged $16-30$ years (mean age, $24 \pm 2.9$ years), participated in this study. The singers had $3-5$ years of formal training experience in Carnatic music. Cortical activities in areas corresponding to attention, discrimination, and memory were tested using P300 analysis, and the tests were performed using the Intelligent Hearing System. Results: The mean P300 amplitude of the singers at the Fz electrode placement site $(5.64 \pm 1.81)$ was significantly higher than that of the non-singers $(3.85 \pm 1.60 ; \mathrm{t}(25)=3.3, p<0.05)$. The amplitude at the $\mathrm{C} z$ electrode placement site in singers $(5.90 \pm 2.18)$ was significantly higher than that in non-singers $(3.46 \pm 1.40 ; t(25)=3.3, p<0.05)$. The amplitude at the Pz electrode placement site in singers (4.94 \pm 1.89$)$ was significantly higher than that in non-singers $(3.57 \pm 1.50 ; \mathrm{t}(25)=3.3$, $\mathrm{p}<0.05)$. Among singers, the mean $\mathrm{P} 300$ amplitude was significantly higher in the $\mathrm{Cz}$ site than the other placement sites, and among non-singers, the mean P300 amplitude was significantly higher in the Fz site than the other placement sites, i.e., music training facilitated enhancement of the P300 amplitude at the Cz site. Conclusions: The findings of this study suggest that more than three years of training in Carnatic singing can enhance neural coding to discriminate subtle differences, leading to enhanced discrimination abilities of the brain, mainly in the generation site corresponding to $\mathrm{Cz}$ electrode placement.

J Audiol Otol 2021;25(3):119-123

KEY WORDS: Cognition · Music therapy · P300 event related potential · Working memory.

\section{Introduction}

Research review suggests that there are numerous studies linking association between music training and development of various cognitive parameters [1-3]. It is assumed that music training enhances central executive function of the discrimination ability, however, there is paucity of empirical data to support this hypothesis [4]. The role of robust cognitive process have been extensively advocated from language acqui-

This is an Open Access article distributed under the terms of the Creative Commons Attribution Non-Commercial License (https://creativecommons.org/licenses/by-nc/4.0/) which permits unrestricted non-commercial use, distribution, and reproduction in any medium, provided the original work is properly cited. sition to the masterful use of language at various levels of life [5]. The role of cognitive skills has been also identified to ensure effective use of language and other higher functions. Furthermore, cognitive approach based speech and language intervention is also extensively used to address a wide range of communication disorders.

Among various event related potential (ERP) components, the $\mathrm{P} 300$ is the most important component for assessing cognitive processes such as attention, working memory (WM), and concentration [6]. P300 scalp distribution is defined as the amplitude change over the midline electrodes $(\mathrm{Fz}, \mathrm{Cz}$, and $\mathrm{Pz}$ ), which typically increases in magnitude from the frontal to parietal electrode sites and is referred to as a P300 
when recorded in response to difficult stimulus contrasts [7]. A multitude of nonpathologic and pathologic subject factors influence the P300. For example, pathologic factors of dementia, depression, and dyslexia affect the P300, as do nonpathologic factors of fatigue, handedness, and personality [8]. The P300 waves varies across experiments, and it is likely that widespread but possibly focal regions contribute to the P300 waves, including hippocampal, sensory-specific cortex, centroparietal cortex, and frontal cortex [9]. The P300 may have multiple intracerebral generators, with the hippocampus and various association areas of the neocortex all contributing to the scalp-recorded potential.

Apart from the behavioral approach, association between music training and cognitive development has been explored at neural level $[10,11]$. Thus, recording of the ERP has been widely accepted as an effective method [12]. The P300 wave is an ERP and it is elicited in the process of decision making. It is considered to be an endogenous potential, as its occurrence links to the specific reaction by an individual not to the physical attributes of a stimulus [13]. Precisely, the P300 is thought to reflect the processes involved in detection of target stimulus [14]. It is usually elicited using the oddball paradigm, in which low-probability target items are mixed with highprobability non-target items [15]. The positive wave in the latency region of $300 \mathrm{~ms}$ are generated from the detection of target signal, which are infrequent (rare), unpredictable (presented randomly), or different (deviant) in some way from the first signal [16]. The response is sometimes referred as P300 because it is observed in the $300 \mathrm{~ms}$ region, and sometimes as the P300 wave, because it forms a third major positive voltage component after auditory late response waves PI and P2 [17].

The P300 response itself is a slow and broad positive peak that may occur between 250 and $800 \mathrm{~ms}$ and may exceed $10 \mu \mathrm{V}$ [18]. The P300 wave is elicited by a task known as the odd-ball paradigm. During this task, a series of one type of frequent stimuli (standard stimulus) is presented along with a different type of non-frequent (target) stimulus [19]. The task of the experimental subject is to react to the presence of target stimulus by a given motor response. If a person attends to target stimuli, P300 is produced and if not then $\mathrm{P} 300$ potentials are not produced.

The duration of music training linking the discrimination ability of cortical areas is highly varying from less than five years, five years to 10 years, and more than 10 years $[2,20]$. The empirical evidences for the role of duration of music training and its subsequent impact on enhancement of discrimination ability are limited. In the present study, it is assumed that shorter duration such as less than five years of music training may enhance the finer cognitive skills such as attention, discrimination, and memory, which are actively used in the learning of music, speech, and language. Furthermore, atleast three years of music training enhances the ability to detect minor changes in the auditory stimulus. Carnatic and Hindusani are the music forms of Inida, based on the availability of singer category of paprticipants in southern part of country Carnatic music was chosen [21].

Thus, primary objective of the study was to investigate which areas of the brain i.e., $\mathrm{Fz}, \mathrm{Cz}$, and $\mathrm{Pz}$ gets more strengthes after exposure of music traing of more than three years. Secondly, the objective was to compare the $\mathrm{P} 300$ response across singers and age matched control group.

\section{Subjects and Methods}

\section{Participants}

A total of 27 individuals categorized into 13 singers of age range between $16-30$ years (mean \pm standard deviation, $23 \pm$ 3.2 years) and 14 non-singers aged range between 16-30 years $(24 \pm 2.9$ years) participated in this study. The participants in the singer group had a minimum of three years of formal Carnatic music learning experience. The participants had a singing history of minimum 5-6 hours of practice in a week. The participants were residence of Mysore district of Karnataka state in India. Written consent was obtained from every participants and the institutional ethical approval was also obtained (13AUD016).

\section{Equipment and stimulus}

The Intelligent Hearing Systems auditory evoked potential system with smart electrophysiology software (IHS 4.3.02 version; Intelligent Hearing Systems, Miami, FL, USA) was used for recording and analyzing P300. The P300 was done by using oddball paradigm, with /da/ as a frequent stimulus and /ga/ as an in-frequent (target) stimulus. Stimulus was presented in right ear at $70 \mathrm{~dB} \mathrm{nHL}$ in alternating polarity with a repetition rate of $1.1 / \mathrm{s}$. Probability of non-target (frequent) and target stimulus was set to $80 \%$ and $20 \%$, respectively. A total of 120 sweeps were presented and $100 \mathrm{~ms}$ prestimulus and $800 \mathrm{~ms}$ post stimulus waveforms were analyzed. Disc electrode was used for recording P300 waveform. Non-inverting electrode placed at $\mathrm{Fz}, \mathrm{Cz}$, and $\mathrm{Pz}$ site, inverting at tip of nose and ground electrode at ipsilateral mastoid.

\section{Procedure}

In the present study, P300 responses were measured, the participants underwent preliminary hearing evaluation before P300 testing. The P300 responses were recorded by instructing the participants to sit in a comfortable reclining chair and were asked to mentally detect the target stimulus /g/. The Fz, 
$\mathrm{Cz}$, and $\mathrm{Pz}$ electrode sites were cleaned with skin preparation gel and the disc electrodes were placed using a conduction paste. Prior to recording P300, an absolute impedance of less than $5 \mathrm{k} \Omega$ and relative impedance of less than $2 \mathrm{k} \Omega$ was ensured. The participants were asked to minimize eye blinks to reduce contamination from alpha activity. The stimulus was presented to right ear. Blocks of stimulus were presented in a series of frequent/non-target (/d/) and infrequent/target $(/ \mathrm{g} /)$ stimulus. The participants were instructed to pay attention to the blocks of stimuli which were presented and were asked to mentally detect only the target stimulus (/g/) during the auditory presentation mode. The discrimination ability of an individual for $/ \mathrm{d} /$ and $/ \mathrm{g} / \mathrm{stimulus}$ was indexed by measuring the P300 potential in $\mu \mathrm{V}$. A total of 120 target sweeps were presented with 350-400 non-target sweep with a probability of $20 \%$ and $80 \%$ for target and non-target sweep respectively. The auto adjusted sweep mode of the instrument minimized the impact of artifact on the study outcome by providing extra target sweeps. That is, every participant received same number of survived sweeps (120) after artifact rejection.

\section{Sample characteristics}

The normality test on the data obtained for the P300 and digit span task was performed for both singer and non-singer groups. The Shapiro-Wilk test was administered and it was observed that the amplitude values of P300 data were normally distributed. Level of significance was tested at 0.05 level.

\section{Results}

The amplitude of P300 was compared between Fz, Pz, and $\mathrm{Cz}$ sites within the group and the P300 response was com- pared for $\mathrm{Fz}, \mathrm{Pz}$, and $\mathrm{Cz}$ sites across the singers and non-singers groups. Analysis of variance test was performed to compare the amplitude of $\mathrm{Fz}, \mathrm{Pz}$, and $\mathrm{Cz}$ sites within the group. For singers, the mean $\mathrm{P} 300$ amplitude at $\mathrm{Fz}$ site $(5.64 \pm 1.81), \mathrm{Cz}$ site $(5.90 \pm 2.18)$, and $\mathrm{Pz}$ site $(4.94 \pm 1.89)$ was compared using Bonferroni multiple comparisons test, a significantly larger P300 amplitude was obtained for Cz site, as compared to Fz and $\mathrm{Pz}$ site in a order $\mathrm{Cz}>\mathrm{Fz}>\mathrm{Pz}$. For non-singers, the mean P300 amplitude at $\mathrm{Fz}$ site $(3.85 \pm 1.60), \mathrm{Cz}$ site (3.46 \pm 1.40$)$, and Pz site $(3.57 \pm 1.50)$ was compared using Bonferroni multiple comparisons test, a significantly larger P300 amplitude was obtained for $\mathrm{Fz}$ site, as compared to $\mathrm{Cz}$ and $\mathrm{Pz}$ site in an order $\mathrm{Fz}>\mathrm{Pz}>\mathrm{Cz}$ (Fig. 1).

Independent sample t-test was performed to compare the amplitude of the $\mathrm{Fz}, \mathrm{Cz}$, and $\mathrm{Pz}$ electrodes between the two groups. For Fz, the mean P300 amplitude of 13 singers (5.64 \pm 1.81) was compared to the 14 participants of the non-singer group (3.85 \pm 1.60$)$, a significantly larger $\mathrm{P} 300$ amplitude $[\mathrm{t}(25)=$ $2.72, p<0.05]$ in singers was obtained. For Cz, the mean P300 amplitude of 13 singers $(5.90 \pm 2.18)$ was compared to the 14 participants of the non-singer group $(3.46 \pm 1.40)$, a significantly larger $\mathrm{P} 300$ amplitude $[\mathrm{t}(25)=3.48, p<0.05]$ was obtained in singers. For Pz, the mean P300 amplitude of 13 singers $(4.94 \pm 1.89)$ was compared to the 14 participants of the nonsinger group $(3.57 \pm 1.50)$, a significantly larger P300 amplitude $[\mathrm{t}(25)=2.08, p<0.05]$ in singers was obtained. Characteristics of P300 waveform obtained in singers and non-singers are displayed in Figs. 2 and 3.

\section{Discussion}

In the present study, the mean P300 amplitude across sing-
Fig. 1. Single plot dipection of $P 300$ amplitude $(\mu \mathrm{V})$ of singers and nonsingers at $\mathrm{Fz}, \mathrm{Cz}$, and $\mathrm{Pz}$ sites.

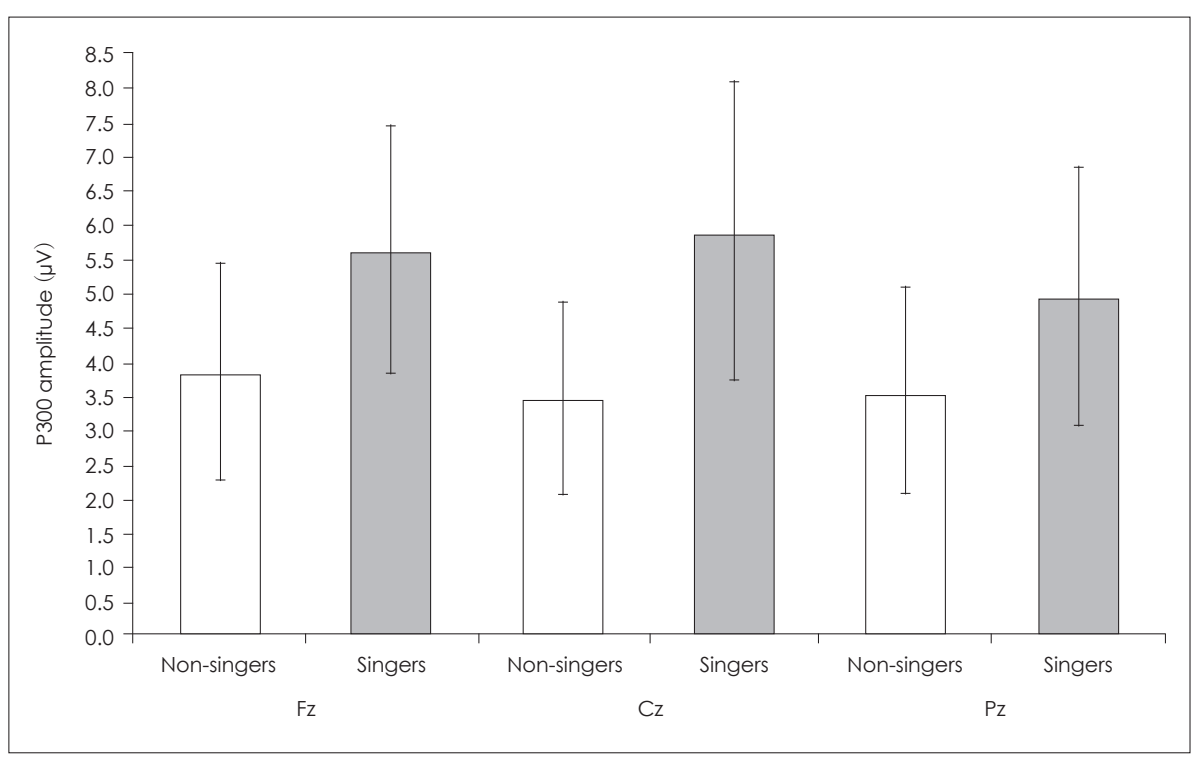




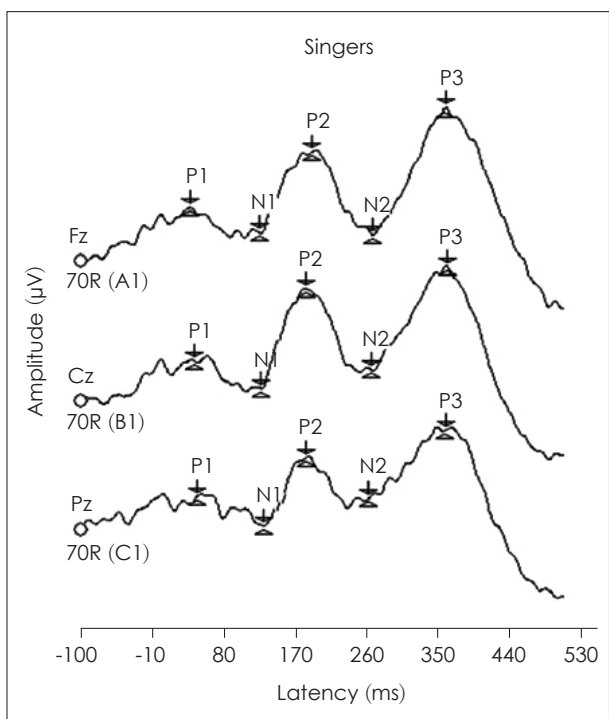

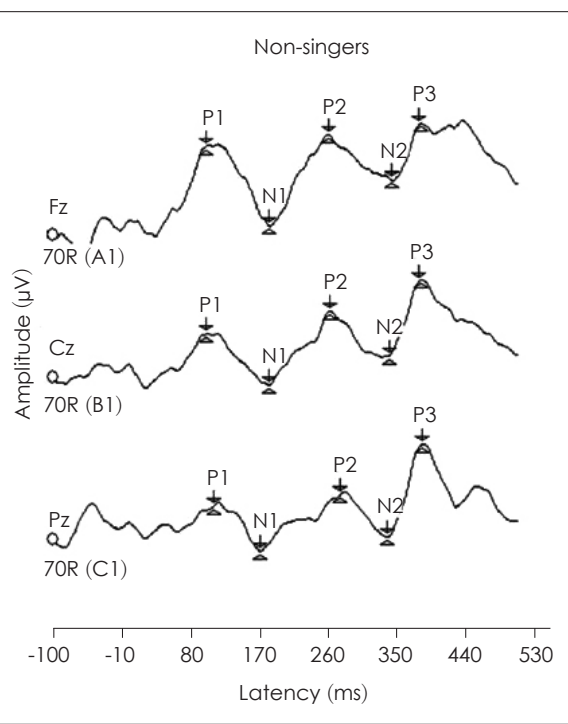

Fig. 2. Characteristics of $P 300$ waveform in singers and non-singers at $\mathrm{Fz}, \mathrm{Cz}$, and $\mathrm{Pz}$ sites.

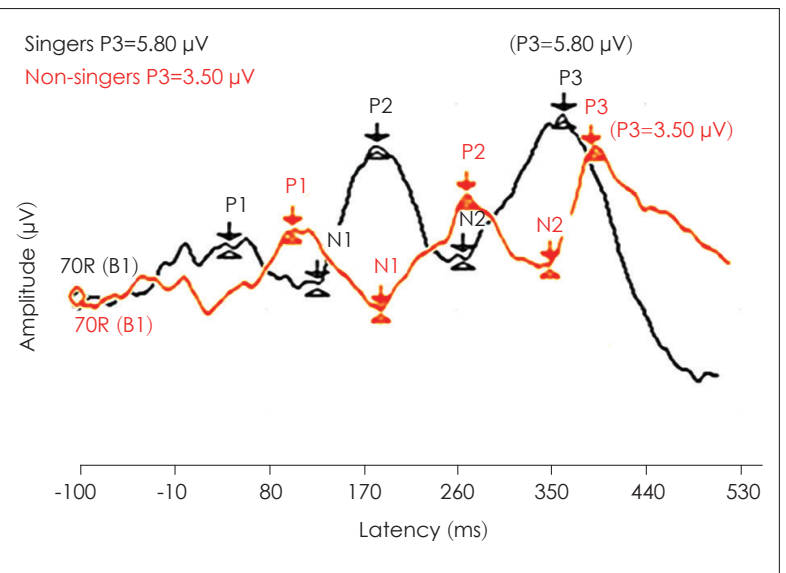

Fig. 3. $P 300$ amplitude $(\mu \mathrm{V})$ in singers and non-singers at $F z, C z$, and $\mathrm{Pz}$ sites.

ers and non-singers was compared at various placement sites, and it was observed that P300 amplitude was significantly higher for singers than the non-singers. These findings suggests the hypothesis of this study that music training induces subtle change in the WM capacity and neural coding of discrimination abilities of the typically developing adult population [22,23]. The role of duration of music training on WM development and discrimination abilities has not been extensively explored. The present study also highlights the notion that even three years of music training is sufficient to induce significant change in the WM capacity and discrimination abilities.

The higher P300 amplitude of the singers group indicates that music training can enhance the ability to detect subtle change in the auditory signals. The enhanced amplitude of the P300 response suggests that the ability of the central executive system of the WM improves with exposure of music training [10]. Hippocampus lobe, sensory-specific cortex, centroparietal cor- tex, and frontal cortex are responsible for the generation of $\mathrm{P} 300$ [9]. The P300 response is likely to have multiple generators, mostly in and around the hippocampus [16]. The site of placement of $\mathrm{Cz}$ electrods is near to the hippocampus, thus, amplitude of $\mathrm{Cz}$ was found better in singers compared to other sites such as Fz and Pz. These findings support that musicians have better hippocampus lobe than non-musicians [24].

The enhanced P300 amplitude of the present study also suggests that the singers allocated more neural resources, reflecting greater sensitivity to the standard and deviant difference in auditory condition $[1,25]$. Music training enforces selective improvements in WM, especially in the central execution which reflects at both neural and behavioral levels [1]. Marked improvement was seen on neural differentiation of the syllables in musicians [26]. Anatomical and neurophysiological evidence of the present study indicates that the formally trained singers showed more efficient neural detection of auditory stimulus which demonstrated superior auditory sensory-memory [27].

Assessment of WM capacity in developing children or the adult neurogenic progressive disorders can be clinically resourceful. In the developing children, adequate growth of various cognitive processes is the prerequisite for speech and language development. Furthermore, assessment of the WM capacity and discrimination abilities can serve as a robust predictor of language and other higher cognitive-communicative skills. The present study justifies the need for development and establishment of a test protocol, specially a ERP:P300 tests for the assessemnt of WM capacity and discrimination abilities.

In conclusion, in the present study, electrophysiological test (P300) was performed on singers and non-singers at various electrode placement sites. Higher P300 amplitude was observed for the singers compared to non-singers for all electrode 
placement sites. Neural generators of $\mathrm{Cz}$ site are more enhanced by music training, as compared to the neural generators of other placement sites. Findings of this study suggest that singers have enhanced higher order processing, which includes their improvised cognitive abilities. The outcome of this study can serve in development of a clinical protocol for assessment of WM capacity in cognitive deficit, developing children and in cognitively declining geriatric population.

\section{Acknowledgments}

The support provided by Amity University Haryana and All India Institute of Speech and hearing Mysuru, Karnataka is acknowledged.

\section{Conflicts of interest}

The authors have no financial conflicts of interest.

\section{Author Contributions}

Conceptualization: Neelesh Benet and Rajalakshmi Krishna. Data curation: Neelesh Benet and Vijay Kumar. Formal analysis: Neelesh Benet and Vijay Kumar. Investigation: Neelesh Benet and Rajalakshmi Krishna. Methodology: all authors. Project administration: Rajalakshmi Krishna and Neelesh Benet. Resources: all authors. Software: Neelesh Benet and Vijay Kumar. Supervision: Rajalakshmi Krishna and Vijay Kumar. Validation: Rajalakshmi Krishna and Neelesh Benet. Visualization: Neelesh Benet and Rajalakshmi Krishna. Writing — original draft: Neelesh Benet and Vijay Kumar. Writing — review \& editing: all authors. Approval of final manuscript: all authors.

\section{ORCID iDs}

Neelesh Benet

Rajalakshmi Krishna

Vijay Kumar

https://orcid.org/0000-0001-6287-7906

https://orcid.org/0000-0003-4501-4265

https://orcid.org/0000-0001-9725-2033

\section{REFERENCES}

1) George EM, Coch D. Music training and working memory: an ERP study. Neuropsychologia 2011;49:1083-94.

2) Bergman Nutley S, Darki F, Klingberg T. Music practice is associated with development of working memory during childhood and adolescence. Front Hum Neurosci 2014;7:926.

3) Brandler S, Rammsayer TH. Differences in mental abilities between musicians and non-musicians. Psychol Music 2003;31:123-38.

4) Forgeard M, Winner E, Norton A, Schlaug G. Practicing a musical instrument in childhood is associated with enhanced verbal ability and nonverbal reasoning. PLoS One 2008;3:e3566.

5) Macnamara J. Cognitive basis of language learning in infants. Psychol Rev 1972;79:1-13.

6) Sowndhararajan K, Kim M, Deepa P, Park SJ, Kim S. Application of the P300 event-related potential in the diagnosis of epilepsy disorder: a review. Sci Pharm 2018;86:10.

7) Squires NK, Squires KC, Hillyard SA. Two varieties of long-latency positive waves evoked by unpredictable auditory stimuli in man. Elec- troencephalogr Clin Neurophysiol 1975;38:387-401.

8) Polich J. Clinical application of the P300 event-related brain potential. Phys Med Rehabil Clin N Am 2004;15:133-61.

9) Picton TW. The P300 wave of the human event-related potential. J Clin Neurophysiol 1992;9:456-79.

10) Morgan HM, Klein C, Boehm SG, Shapiro KL, Linden DE. Working memory load for faces modulates P300, N170, and N250r. J Cogn Neurosci 2008;20:989-1002.

11) Kumar V, Sanju HK. Development of chunk size and capacity as a predictor of working memory in hindi speaking typically developing children. Open Access J Neurol Neurosurg 2017;6:27-30.

12) Polich J. Neuropsychology of P300. In: The Oxford Handbook of Event-Related Potential Components (eds. Luck SJ, Kappenman ES). Oxford: Oxford University Press;2012. p.159-88.

13) Pritchard WS. Psychophysiology of P300. Psychol Bull 1981;89:50640.

14) Azizian A, Freitas AL, Watson TD, Squires NK. Electrophysiological correlates of categorization: P300 amplitude as index of target similarity. Biol Psychol 2006;71:278-88.

15) Katayama J, Polich J. Auditory and visual P300 topography from a 3 stimulus paradigm. Clin Neurophysiol 1999;110:463-8.

16) Hall JW. New handbook of auditory evoked responses. Boston: Pearson;2007.

17) Musiek FE, Froke R, Weihing J. The auditory P300 at or near threshold. J Am Acad Audiol 2005;16:698-707.

18) Polich J. Normal variation of P300 from auditory stimuli. Electroencephalogr Clin Neurophysiol 1986;65:236-40.

19) Mertens R, Polich J. P300 from a single-stimulus paradigm: passive versus active tasks and stimulus modality. Electroencephalogr Clin Neurophysiol 1997;104:488-97.

20) Benassi-Werke ME, Queiroz M, Araújo RS, Bueno OF, Oliveira MG. Musicians' working memory for tones, words, and pseudowords. Q J Exp Psychol (Hove) 2012;65:1161-71.

21) Ranjani H, Arthi S, Sreenivas T. Carnatic music analysis: Shadja, swara identification and raga verification in alapana using stochastic models. In: 2011 IEEE Workshop on Applications of Signal Processing to Audio and Acoustics (WASPAA) (eds. Ranjani H, Arthi S, Sreenivas T). New Paltz: IEEE;2011. p.29-32.

22) Anaya EM, Pisoni DB, Kronenberger WG. Long-term musical experience and auditory and visual perceptual abilities under adverse conditions. J Acoust Soc Am 2016;140:2074-81.

23) Rabelo CM, Neves-Lobo IF, Rocha-Muniz CN, Ubiali T, Schochat E. Cortical inhibition effect in musicians and non-musicians using P300 with and without contralateral stimulation. Braz J Otorhinolaryngol 2015;81:63-70.

24) Gaser C, Schlaug G. Brain structures differ between musicians and non-musicians. J Neurosci 2003;23:9240-5.

25) Parbery-Clark A, Tierney A, Strait DL, Kraus N. Musicians have fine-tuned neural distinction of speech syllables. Neuroscience 2012; 219:111-9.

26) Kraus N, Slater J, Thompson EC, Hornickel J, Strait DL, Nicol T, et al. Music enrichment programs improve the neural encoding of speech in at-risk children. J Neurosci 2014;34:11913-8.

27) Schneider P, Scherg M, Dosch HG, Specht HJ, Gutschalk A, Rupp A. Morphology of Heschl's gyrus reflects enhanced activation in the auditory cortex of musicians. Nat Neurosci 2002;5:688-94. 